\title{
Radiologic findings of epiploic appendagitis
}

\section{Cigdem Oztunali, Taylan Kara}

Eskisehir Osmangazi University Medical Faculty, Department of Radiology, Eskisehir, Turkey

\begin{abstract}
The aim of this case report is to present typical US and CT features of epiploic appendagitis, characterized by the ischemia and inflammation of the epiploic appendices of the colon.

A 44 year old man was admitted with a localized pain to umblical region. Physical examination revealed rebound tenderness. US examination showed a hyperechoic mass with a hypoechoic rim posterior to abdominal wall and CT features confirmed the diagnosis of epiploic appendagitis. The case was managed conservatively and had a self limited course.

Keywords: epiploic appendagitis, ultrasonography, computed tomography
\end{abstract}

\section{Introduction}

Epiploic appendagitis is a rare, self limited condition characterized by the ischemia and inflammation of the epiploic appendages. It is mostly manifested as a localized, severe pain, usually in the right or left lower quadrant and it can mimic acute diverticulitis or appendicitis [1-4]. Knowledge of the radiologic features of this entity is important for patient management and can avoid unnecessary surgery. The aim of this report is to present clinical and radiological findings of a case of epiploic appendagitis.

\section{Case Report}

A 44-year-old man presented to emergency department with progressively increasing acute abdominal pain and nausea. Physical examination revealed re-

Received 26.09.2012 Accepted 15.10.2012

Med Ultrason

2013, Vol. 15, No 1, 71-72

Corresponding author: Taylan Kara MD

Eskisehir Osmangazi University Medical

Faculty, Department of Radiology

26000, Eskisehir, Turkey.

Phone: +90 (506) 3663631

E-mail: taylankara@gmail.com bound tenderness in the umblical region. The patient was afebrile and had normal bowel movements. His rectal examination and laboratory findings were unremarkable. Abdominal ultrasonography (US) of the liver and biliary tract was normal. Under the site of patient's maximum tenderness, there was a $2.5 \times 1.5 \mathrm{~cm}$ hyperechoic oval mass posterior to abdominal wall (fig 1 ). The mass had a hypoechoic rim and there was no internal flow with color Doppler imaging. With the suspicion of epiploic appendagitis, a contrast enhanced abdominal computed tomography (CT) examination was performed after an intravenous enjection of $100 \mathrm{ml}$ nonionic contrast material with an iodine content of $300 \mathrm{mg} /$ $\mathrm{ml}$ at a rate of $4 \mathrm{ml} / \mathrm{s}$ (Omnipaque 300, Nycomed Amersham, New York, USA). A portal venous phase scan was obtained after a total scan delay of $80 \mathrm{~s}$, from diaphragm to the symphysis pubis. Scanning parameters included $120 \mathrm{kVp}, 250 \mathrm{~mA}$ and a slice thickness of 0.5 $\mathrm{mm}$. CT demonstrated an oval lesion of fat density, adjacent to the anterior wall of the right side of the transverse colon (fig 2). Mesenteric fat planes surrounding the lesion were increased in density. The lesion showed peripheral contrast enhacement. A diagnosis of epiploic appendagitis was made according to US and CT findings. After a course of 5-day antibiotic therapy patient's clinical symptoms remitted. 


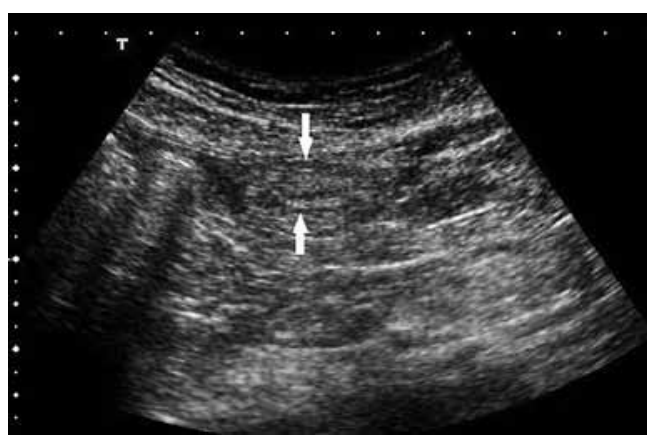

Fig 1. Hyperechoic oval mass posterior to abdominal wall seen on ultrasonography (arrows)

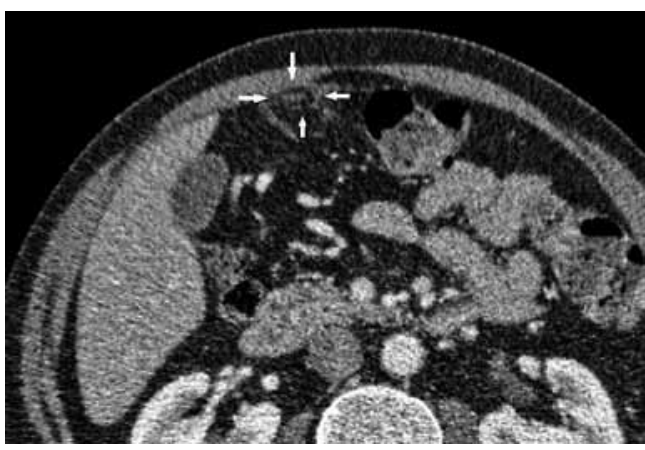

Fig 2. Contrast enhanced axial abdomianl CT demonstrates an oval lesion of fat density and increased density in surrounding fat planes (arrows).

\section{Discussion}

Epiploic appendages are $0.5-5 \mathrm{~cm}$ long and $1-2 \mathrm{~cm}$ thick, serosa-covered, fatty pouchings originating from the antimesenteric surface of the colon. They are arranged in two rows paralleling the tenia coli and their number and size increase in the lower quadrants $[1,3]$. The function of these appendages remains unclear but a cushioning function for the colon and a role in immune response and absorbtion have been proposed [5]. Each appendage is attached to the colon with a stalk containing one or two arterioles and a venule. Spontaneous kinking or twisting of this vascular stalk or the thrombosis of the draining venule causes ischemia and inflammation resulting in the primary form of these appendages, known as primary epiploic appendagitis. Secondary form of appendagitis is caused by any other inflammatory process involving colon, such as diverticulitis or appendicitis [2].

Epiploic appendagitis mostly manifests in the 4th and 5th decades of life with a male predominance $[3,5]$.
Clinically, patients usually have a localized, sharp pain in the lower quadrants without any associated symptoms such as vomiting or diarrhea. Laboratory findings are unremarkable in most of the cases although a low grade leukocytosis and an increase in $\mathrm{C}$ reactive protein levels have been reported $[1,6]$. CT, US and MRI can aid in the diagnosis of epiploic appendagitis. US typically shows a non-compressible hyperechoic, oval mass with a hypoechoic rim and no central flow on color Doppler imaging under the point of maximum tenderness. CT demostrates a fat-density lesion next to the colon with surrounding mesenteric fat stranding and sometimes a contrast-enhanced rim. A hyperdense linear area can be seen centrally, representing the thrombosed venule $[2,3,7]$. The differential diagnosis of a fatty lesion in a patient with abdominal pain includes diverticulitis, appendicitis, omental infarction and mesenteric panniculitis $[2,3]$.

In conclusion, epiploic appendagitis is a rare cause of acute abdomen and should be suspected in a patient with localised pain and tenderness and no associated symptoms. Recognition of radiologic findings of this entity is important because it is managed medically and has a self- limited course. CT features of epiploic appendagitis is well known and recognition of its typical US findings can also be helpful in the diagnosis.

\section{References}

1. Sand M, Gelos M, Bechara FG, et al. Epiploic appendagitis - clinical characteristics of an uncommon surgical diagnosis. BMC Surg 2007; 7: 11 .

2. Almeida AT, Melão L, Viamonte B, Cunha R, Pereira JM. Epiploic appendagitis: an entity frequently unknown to clinicians - diagnostic imaging, pitfalls, and look-alikes. AJR Am J Roentgenol 2009; 193: 1243-1251.

3. Singh AK, Gervais DA, Hahn PF, Sagar P, Mueller PR, Novelline RA. Acute epiploic appendagitis and its mimics. Radiographics 2005; 25: 1521-1534.

4. Pereira JM, Sirlin CB, Pinto PS, Jeffrey RB, Stella DL, Casola G. Disproportionate fat stranding: a helpful CT sign in patients with acute abdominal pain. Radiographics 2004; 24: 703-715.

5. Uslu Tutar N, Ozgül E, Oğuz D, Cakir B, Tarhan NC, Coşkun M. An uncommon cause of acute abdomen - epiploic appendagitis: CT findings. Turk J Gastroenterol 2007; 18: $107-110$.

6. Uehara R, Isomoto H, Yamaguchi N, et al. Epiploic appendagitis in a 27-year-old man. Med Sci Monit 2011; 17: CS113-115.

7. Singh AK, Gervais DA, Hahn PF, Rhea J, Mueller PR. CT appearance of acute appendagitis. AJR Am J Roentgenol 2004; 183: 1303-1307. 